takes the central part of a glanders nodule, that lesion also presents a close histological affinity with the granuloma of actinomycosis. In that also the foundation of the lesion, and the part of it which immediately surrounds the parasites, is almost entirely composed of leucocytes. There is also observable the same tendency to an early arrest of the growth or extension of the lesion, while the fibroblasts or other fixed tissue cells begin to construct a fibrous or epithelioid zone around the leucocyte centre.

The lesions of farcy are at the outset histologically identical with the solid lesion which is usually termed a glanders nodule. There is the same migration of leucocytes, and the same disappearance of the normal tissue elements from the parts where the leucocytes have congregated. But what is only exceptional in the case of the pulmonary nodules - the progressive liquefaction of the tissues around the initial lesion-is the rule in the lesions of farcy, which thus become converted into actual abscesses. The subcutaneous tissue, perhaps in consequence of its richness in lymphatic channels, appears to be a specially favourable soil for the glanders bacillus, and one in which its tendency to progressively invade the tissue around the initial lesion is less easily held in check by the leucocytes. Here also the leucocytes are liable to chromatotexis, but that is much less complete than in the centre of the solid glanders nodule. As is well known, the pus which is discharged from a farcy bud is ordinarily less opaque and creamy than that which is formed under the action of the common pyogenic bacteria. Its greater viscidity and slighter opacity are apparently due to an unusual proportion of albuminous material and a smaller number of leucocytes or pus corpuscles. There are, however, farcy lesions in which the pus has nothing in its naked eye characters to distinguish it from the pus of what may be called ordinary abscesses.

\title{
SOME DISEASES COMPLICATING RINDERPEST AMONG CATTLE OF INDIA.
}

\section{By J. D. E. Holmes, M.A., M.R.C.V.S., Capt., I.C.V.D., Assistant Bacteriologist, Muktesar, India.}

RINDERPEST is a disease which runs a very regular course, and one of which the diagnosis usually presents little difficulty. Among the cattle of India the degree of susceptibility to this disease varies very considerably. In some districts in the Plains, where the ravages of rinderpest are of frequent occurrence, the percentage of animals resistant to the disease is very high, and in many outbreaks a mortality of about 25 per cent. only has been recorded. This immunity may be ascribed to such causes as " hereditary influence," protection afforded by a previous attack of the disease, and survival of the fittest. In other parts of the country, especially in the mountainous tracts, where traffic is less, and cattle are not exposed to the danger of infection in fairs and markets, rinderpest is almost unknown, but when it does make an appearance it carries off about 90 per cent. of animals attacked. In the first class of animals, namely, the bulls and cows ordinarily met with in the villages of the plains, the course of the 
disease is somewhat modified, the stages are more prolonged, the symptoms are less marked, and recovery is frequent.

In the cattle of the hill breed the disease takes a very acute and fatal course. In inoculated cases the three stages of the malady are well marked, and each is usually of about three days' duration. These are (I) the incubative period, (2) the febrile stage, when all the lesions can be observed, and (3) the stage of diarrhœea, on the second day of which the temperature rapidly falls, and the animal dies on the eighth or ninth day.

The post-mortem appearances in every case show a striking similarity, and in this country secondary lung lesions are rarely found. The pathological changes throughout the whole mucous membrane of the alimentary canal present a picture which is not simulated by any other disease. There is, perhaps, no solitary lesion upon the existence of which a certain diagnosis can be founded. Before general exfoliation of the mucous membrane takes place, the pin-point ulcers on the surface of the tongue, gums, and pharynx are typical. The formation of vesicles and ulcers on the mucosa of the gall bladder is stated by some authors to be characteristic of rinderpest alone. These lesions, however, have been observed in fatal cases of Texas fever, and I have seen the same appearances caused by the presence of distomata. The congested condition of the Peyer's patches often exists in other inflammatory affections of the bowels, but in no other disease of cattle are these glands found to be covered with necrotic material as in rinderpest.

The typical case of rinderpest is one which it is not difficult to recognise, and anyone who has had an experience of one or more outbreaks may consider that there is little more to be learnt of the clinical or post-mortem symptoms.

There are, however, many variations in both, due not to the disease itself but to other pathological conditions playing a secondary part. W'hen an isolated or individual case of such a kind is met with it is very frequently a matter of difficulty to form a decided opinion. I have seen many animals die from the disease which during life showed no characteristic symptoms. At the post-mortem all the typical necrotic changes in the alimentary canal were marked. Many cases show a rise of temperature only, and after death there is nothing to be found which might not be due to gastro-enteritis.

Many causes may be held to account for these phenomena: a variation in the virulence of the contagium, a difference in the resistant power of the cells of the body, or the still less understood term of individual idiosyncracy.

There is no doubt that these and other unknown factors cause many deviations in the typical course of a disease.

It has, however, been observed in this and in other Laboratories where anti-rinderpest serum is prepared that certain other diseases frequently run a course concurrent with rinderpest, and in many respects either intensify or modify the effect of that disease. The recognition of such secondary affections, and an understanding of the part played by them, is of much importance both in the preparation of serum and in its practical application.

Some of these diseases I have had an opportunity of studying at the Bacteriological Laboratory, Muktesar, among the animals experi- 
mentally inoculated with rinderpest virus, for the purpose of preparing the anti-serum, and for testing the potency of such serum before issuing.

\section{PIROPLASMOSIS.}

This scourge of cattle, known under many names (Texas fever, redwater, bovine malaria, tristeza, etc.), is one which has been recognised in almost every country and fully described by many authors.

In India the disease is said to have been first clinically observed by Hallen in I87 I, but from later publications by Queripel, Gunn, and others, and from regulations laid down concerning the examination of horses imported from Australia to prevent ticks being brought in, the existence of Texas fever in the country does not seem to have been accepted as proved.

Queripe ${ }^{1}$ discussed the question, and was inclined to believe that the disease did exist in India, as the symptoms of "red urine" and "wasting" were well known to zemindars. At the same time, he advised that horses landed from Australia should be examined for ticks.

Gunn 2 stated that all microscopical examinations of blood had failed to demonstrate the micro-organism of Texas fever, and expressed the view that the ticks of India were not contaminated with the parasite of the disease. He concluded that the disease did not exist in Inclia, and that all precautions should be taken to exclude it from the country.

In I 898 Raymond reported the presence of piroplasma bigeminum in cattle of India.

The piroplasma was demonstrated in the blood of the cattle of this country by Stockman ${ }^{3}$ in 1903 , while conducting some experiments relative to the degree of susceptibility to rinderpest among breeds of cattle in the Madras Presidency. Ten out of twenty-three cattle inoculated with rinderpest developed redwater.

The typical pear-shaped bodies are found in the red cells of affected animals. The disease is apparently the same as observed in other countries, and is spread by means of ticks. At the present time we are aware that the protozoa are harboured in the blood of a large percentage of cattle, but we have no accurate knowledge of the extent to which the disease by itself affects animals. Information collected from native owners of cattle shows that a disease recognised by the symptoms of red urine is known to them, and that at times it causes considerable loss. During last year Captain Walker, I.C.V.D., ${ }^{4}$ reported an outbreak of Texas fever where eight animals died.

In the blood of many cattle apparently healthy and in good condition, the small coccus-like bodies described by Smith and Kilborne have been found in the red cells, while a careful and extended search did not reveal any other form of the parasite. It is a significant fact that the pear-shaped bodies and bacillary forms characteristic of the piroplasma bigeminum have been found only in the blood of cattle after they have been inoculated with virulent rinderpest blood.

1 “Agricultural Ledger Series," No. 2 of 1897.

" "Agricultural Ledger Series," No. 2 of 1899.

" "Veterinary Record," 11th April 1903.

Report of Civil Veterinary Department, Punjaub, 1903. 
This seems to point to the conclusion that the cattle of India have for many years had this disease among them, and that a large percentage of animals now possess an immunity sufficient to protect them until this resistant power has been reduced by an attack of another disease. It would also seem as if rinderpest more than any other disease broke down this natural resistance to piroplasmosis. I have made many examinations of the blood of bulls whose vitality had been lowered by an attack of anthrax or black-quarter, or by large subcutaneous injections of virulent cultures of these diseases; also of cattle dying from gastro-enteritis, pneumonia, and other affections, but have not in such cases found the blood infected with the malarial organism.

In other countries it has been noted that cattle inhabiting a region over 5000 feet high do not suffer from malaria, and the reason ascribed is that ticks do not live at this altitude. This is not the case in India. In the Kumaon tract of the Himalayas, at a height considerably over 7000 feet, the cattle are infested with ticks, and I have found a larger percentage of these animals affected with piroplasms than among cattle of plains breed. The parasite found in the blood of these hill bulls presents certain peculiarities.

It is generally seen in form of a spore, ring, rod, or leaf, with a chromatin dot at the apex. I have not been able to find any of the typical pear-shaped forms, and I have not observed the bloodcoloured urine which is usually a clinical symptom of the disease in cattle of the plains. In hill cattle when the piroplasma appears numerous in the blood, during the course or after recovery from a modified attack of rinderpest, a fatal issue almost invariably occurs. In plains cattle the complication has not, as a rule, so rapid and fatal an effect; some cases recover, and the micro-organisms after a time disappear from the blood. It is a question whether, when an animall becomes infected with piroplasms after a subcutaneous injection with virulent rinderpest material, the organism was introduced with the inoculated blood, or had been previously lying dormant in the body. I am of opinion that the latter is more frequently the correct view. In many instances I have examined the virulent blood before inoculation without being able to find any trace of organism, and subsequently the piroplasms have appeared in large numbers in the blood of the injected bull. Also, I have many times injected, both subcutaneously and intravenously, fresh blood containing numerous parasites into a healthy bull, and in no instance have I been able to produce the disease.

As a complication of rinderpest, piroplasmosis appears from five to fifteen days after the inoculation of virulent blood. The only clinical symptoms which at first give a clue to the presence of the disease are, fluctuating temperature, with considerable variation between the morning and evening record, an anæmic condition, and an apparent relapse in animals which are recovering from an attack of rinderpest.

The following cases show the general course of the disease :-

Bull No. I.-Plains breed. Received a simultaneous injection of serum and virulent blood on 28 th March, with the object of immunising him for the preparation of serum. He had a high temperature reaction, with the appearance of vesicles and ulcers on the tongue and buccal membrane. On I 2 th 
April the temperature had returned to normal and the animal was feeding well. On $15^{\text {th }}$ April, the temperature again suddenly rose to $40^{\circ} \mathrm{C}$., and from this date fever was continuous, with a difference of $2^{\circ}$ to $3^{\circ} \mathrm{C}$., between morning and evening temperature. From the appearance of fever the blood was examined daily. Numerous pear-shaped organisms were observed in the red cells. The parasites after a week gradually disappeared, until only a few coccus-like forms could be found. About the end of April the bull was in good health again. He was subsequently injected with $6000 \mathrm{cc}$. of virulent rinderpest blood and afterwards frequently bled for serum, but did not again develop any symptoms of illness.

Bull No. II.-Plains breed. Was injected by the simuluaneous method on the same date as bull No. I. He had a severe attack of rinderpest lasting seven days. About the tenth day the lesions were healing and the bull was recovering, when the temperature again rose to $39^{\circ} 5^{\circ} \mathrm{C}$., and intermittent fever continued for some days. The blood was examined, and about 30 per cent. of the red cells were found to contain the piroplasms. The animal became daily more emaciated, fed little, coat staring, urıne blood-coloured, diarrhoea constant. The condition became worse, and the bull died on the twenty-fifth day.

At the post-mortem the yellow connective-tissue ecchymoses and extravasations, enlarged and softened spleen, and other lesions characteristic of Texas fever, were marked.

In the cases observed of bulls of hill breeds affected with piroplasmosis in conjunction with rinderpest, the symptoms were much the same, with the exception that the fluctuating temperature and the piroplasms in the blood appeared at an earlier date, and the disease ran a more rapid and fatal course.

Bull No. 1II.-Hill breed. Received a simultaneous injection of serum and virulent blood. On the fourth day the evening temperature was $40^{\circ} 2^{\circ} \mathrm{C}$. No symptoms of rinderpest appeared, but fever continued, with $2^{\circ}$ to $3^{\circ} \mathrm{C}$. variation between morning and evening temperature. There was a serous discharge from the nostrils, and the mucous membrane was of a yellowish colour. The animal fed little, continually lying down and at times struggling. Died on the ninth day. The blood was examined on the fifth day, and numerous piroplasms were found. They were of small size, the majority in the shape of a leaf with a chromatin dot at the point, but many were in the form of a ring and others were rod-shaped. Before death the micro-organisms increased somewhat in size and became more numerous. No pear-shaped forms were found. At the post-mortem no lesions of rinderpest were observed. The connective-tissue and mucous membranes were of a yellow colour, and there were small sub-capsular hæmorrhages in the spleen and liver. The spleen was enlarged and softer than normal. Liver engorged. Ecchymoses on surface of intestines, with areas of deep congestion.

Bull No. IV. - Was inoculated in similar method as above. $\mathrm{He}$ had a slight attack of rinderpest, and was recovering, when, on the twelfth day, the temperature again became high, and it remained fluctuating for some days. The condition of the animal each day became worse ; appetite failed. anæmia was marked, diarrhoea set in, and death occurred on the nineteenth day after inoculation. The blood was examined on the twe'fth day and piroplasms were found similar to those seen in the blood of bul No. III. They became slightly larger and much more numerous as the disease advanced. At the post-mortem scars of healed ulcers in the small intestines, pitted Peyer's patches, and other signs of a recent recovery from rinderpest were noticed, together with the characteristic appearance of death from Texas fever. 
Piroplasmosis as a complication of rinderpest has been found at this laboratory to be a cause of considerable loss and trouble. At times it carries off bulls which have been immunised, and which have received a large injection of virulent blood for the preparation of serum. Among the animals inoculated with virulent material for purpose of producing the disease and obtaining blood for injecting serum-making bulls, piroplasmosis frequently caused a variation in the temperature curve and ordinary course of rinderpest. Such animals are not considered suitable for bleeding, and are wasted.

When the potency of a bulk of anti-rinderpest serum is being tested by inoculating a number of bulls with virulent blood and varying doses of serum, the appearance of Texas fever increases the difficulty of ascertaining the amount of serum required to protect against rinderpest, and necessitates the repetition of the experiment.

In the practical use of the serum in outbreaks of rinderpest it is probable that Texas fever arising as a secondary disease may play an important part, and may account for a certain percentage of deaths. Up to the present no observations in this point have been recorded in India.

TRIPANOSOMIASIS AMONG CATTLE OF INIIA.

In a communication which appeared in the preceding number of this Journal (p. 209), a trypanosoma found in a smear preparation taken from the spleen of a hill bull wds described by Capt. Durrant, A.V.D., and myself. As recorded in the above paper, the bull had been used for the purpose of testing the potency of a bulk of antirinderpest serum. He had a slight attack of the disease, from which he was recovering when symptoms of a secondary disease became manifest : diarrhoea, fluctuating temperature, loss of appetite, staring coat, anæmia, resulting in death.

Since the first discovery of this trypanosoma, I have met with many more cases of trypanosomiasis among the cattle of hill breed kept at this Laboratory for experimental purposes, and consequently have had an opportunity of a fuller study of the parasite. In every instance in which the disease has been observed, it has made its appearance secondarily to an attack of rinderpest, or after the animal has been inoculated with a large quantity of virulent blood in order to hyper-immunise for the preparation of serum. The following are the notes of three cases:-

Bull No. I.-Hill breed. Was immunised by a simultaneous injection of virulent rinderpest blood and serum. He had a slight attack evidenced by temperature reaction only. Fourteen days after the first inoculation he was injected subcutaneously with $2500 \mathrm{cc}$. of virulent blood for the purpose of hyper-immunising. This was followed by a rise of temperature, which remained near $40^{\circ} \mathrm{C}$. for six days and then returned to normal. After three days the temperature again reached $4 \mathrm{I}^{\circ} \mathrm{C}$, and stood at that for eight days. On the ninth day the temperature rapidly fell, and the animal died the following night. During the last ten days the bull ate little, became weak and emaciated, and was continually lying down. The last day the animal struggled convulsively; some serous fluid flowed from the nostrils; mucus and blood from rectum. On the appearance of secondary fever the blood was examined and trypanosomata found. On the first day of observation 
there were four parasites in a field, but the number daily increased, and at the time of death they numbered thirty in a field.

Bull No. II.-Hill breed. Was used for the purpose of serum-testing. At the time of inoculation he was in good condition. He had a fairly severe attack of rinderpest, which lasted about twelve days. The lesions of this disease healed, and the temperature returned to normal. The bull, however, remained thin, did not recover its former strensth, and suffered from diarrhoea. The temperature was irregular, and frequently rose above normal. The blood was examined and trypanosomata discovered. They were not numerous, varying from one to three in a field. The animal died a month after the date of inoculation. At the post-mortem of this bull there were no marked lesions; the carcase was emaciated, the blood watery, the spleen somewhat enlarged and soft, and petechial submucous hæmorrhages in small and large intestines.

Bull No. III.-Hill breed. Was hyper-immunised for serum-making, and received a subcutaneous injection of $3000 \mathrm{cc}$. virulent rinderpest blood. This was followed by a rise of temperature, which remained above $40^{\circ} \mathrm{C}$. for some days, after which the chart showed a very fluctuating curve, with a difference of $2^{\circ}$ to $3^{\circ} \mathrm{C}$. between the morning and evening record. The blood was examined for piroplasms and found to contain trypanosomes, two in a field. After several days the temperature became normal, the trypanasomes disappeared, and the bull recovered.

My further observation of the trypanosomes has shown that the parasites which were first noticed, and which were taken from the spleen, were evidently immature forms, and that the description then given differs very considerably from that of the mature flagellate form found in the blood stream.

The full-grown trypanosomes are much longer than the surra parasites, and are probably the longest yet described. The largest depicted in the accompanying Plate measured $9 \mathrm{I} \mu$ in length. The average length is from 60 to $80 \mu$. The breadth is from $2 \mu$ to $4 \mu$, and in some cases as much as $6 \mu$; the latter forms were not typical, and secmed to be undergoing change. The posterior extremity is very fine and elongated, measuring from centrosome to end Io to I $7 \mu$. The flagellum is well developed, and measures from i $5 \mu$ to $25 \mu$. In the younger forms the centrosome is situated very close to the nucleus. In the blood taken from two infected bulls I found peculiar developing forms bearing a striking resemblance to the crescents of human tertian fever. These are large crescent-shaped bodies staining blue and containing numerous chromatin granules.

As I have stated above, the disease was in every instance found in cattle of hill breed which had been treated with rinderpest virulent blood. Up to the present I have not been able to find the trypanosoma in the blood of cattle of plains breed or in uninoculated hill cattle. The apparent immunity of plains cattle may be due to the fact that these are all specially selected cattle, in good condition. This is not the case with the hill bulls; they are of all kinds and conditions, and many are emaciated and half-starved animals. The relation between the rinderpest inoculation and the appearance of the trypanosoma appears to me similar to that observed in piroplasmosis of Indian cattle. These animals, more especially those of certain districts, have probably been for many years subject to the attack of these parasites, and now possess a partial immunity. The disease lies dormant in the body until the normal resistant power of the cells 
has been lowered from some cause, such as an attack of rinderpest. The parasites then develop in the system, exercise a certain amount of harmful effect, and assist the primary disease to a fatal issue.

There is another way in which the appearance of the trypanosoma after rinderpest inoculation may be accounted for. It is possible that the trypanosoma may be conveyed from an infected to a healthy animal with the rinderpest virulent blood. This latter view is less probable. The plains cattle which have been injected with a large dose of virulent blood from hill cattle have not become infected with the trypanosoma; neither have I been able to convey the disease to any experimental animals by means of either subcutaneous or intravenous injections of blood containing numerous trypanosomes. The disease is frequently associated with piroplasmosis.

In the blood of the bull from which the trypanosomes shown in Plate VIII. were taken about 50 per cent. of the red cells were invaded by small piroplasmata. Some cells contained four parasites. This case ended fatally. The disease does not show itself by any distinctive clinical symptoms. In most of the animals in whose blood the trypanosomes were found the existence of the disease was not suspected until the blood was examined. In come instances attention was drawn to these bulls by their poor and anæmic condition. In other cases the bulls at first were in apparent health, but their daily chart showed a fluctuating temperature, with an abnormal variation between the morning and eveningr record.

Only a few cases ended in death, and these bulls were in debilitated condition. In others the trypanosomes were found for several days in the blood, never very numerous, seldom above five in the field. They subsequently disappeared, and were not discovered again, though the blood was examined daily for five to six weeks.

The following experimental inoculations were carried out, but I failed to transmit the disease in so far that the trypanosomata was never found in the blood of the inoculated animals :-

Pony No. I.--Inoculated subcutaneously with $25 \mathrm{cc}$. defibrinated blood from Bull No. 3276 . In the blood used from Bull No. 3276 there were four parasites in a field.

Pony No. II.-Received intravenously $5 \circ \mathrm{cc}$. citrated blood from Bull No. 3025. In this blood there were six trypanosomes in a field.

Bull No. I. (Plains breed) ? Were inoculated subcutaneously with $25 \mathrm{cc}$.

Bull No. II. (Hill breed). $f$ defibrinated blood from Bull No. 3276 .

Bull No. III. (Plains). ? Were inoculated intravenously with $50 \mathrm{cc}$. citrated Bull No. IV. (Hill). $\}$ blood from Bull No. 3025 .

Rabbit No. I.-Received 5 cc. defibrinated blood from Bull No. 3276 .

Rabbit No. II.- Was inoculated intravenously with $2 \mathrm{cc}$. citrated blood from Bull No. 3025 .

\section{ECHINOCOCCUS IDSEASE.}

This clisease is very prevalent among the cattle of India. Pease collected statistics, and found that, among the cattle slaughtered for food in Lahore, 8o per cent. were affected to a greater or less degree. He states from his own experience that hydatid disease is a common cause of death and loss of efficiency among cattle and camels. The prevalence of the disease is due to the number of pariah dogs and jackals, and the defective method of the disposal of the carcases of 
dead animals. At the Laboratory I have noticed that bovines badly affected with hydatids succumb very quickly to an attack of rinderpest. Frequently, after having been inoculated, the animal dies in the incubative stage, before the lesions of rinderpest have appeared. In other cases the temperature does not rise on the third or fourth day, but continues abnormally low, gradually falling until death results on the fifth to seventh day.

In these animals the course of rinderpest is always erratic, and the characteristic symptoms either do not present themselves at all or appear irregularly. At the post-mortem the lesions of rinderpest are rarely well marked, and at times altogether absent.

Bull No. I. Hill breed.--Received a simultaneous injection for the purpose of testing serum. On the second day the morning temperature was $40^{\circ} 4^{\circ} \mathrm{C}$., fell below normal on the following day, and remained low. The animal died the next day. There were no other symptoms of illness. The lungs were crammed with cysts, varying in size from a cricket ball to a marble. The liver was also affected, and the spleen contained one cyst about the size of a cocoanut. The mucous membrane of the small intestines was bile-stained and covered with mucus. No lesions of rinderpest were noted.

Bull No. II. Hill breed. - Was inoculated with virulent blood as a control. The temperature remained normal during the first four days. On the fifth it reached $40.5^{\circ} \mathrm{C}$., and on the next day fell to $36.3^{\circ} \mathrm{C}$, the bull dying on the following day. The lungs and liver were the seat of numerous cysts. The gall-bladder was absent, and in its place was a small cyst the size of a marble containing clear fluid. Another cyst the size of a pea-nut was situated on the border of the central flap of the tricuspid valve of the heart. There were a few vesicles on the gums and under the tongue; the intestines showed slight congestion, but there were no further lesions of rinderpest.

Bull No. III. Hill breed.--Was used for serum testing purpose, and received a simultaneous injection. On the second and third days the temperature was $39.6^{\circ} \mathrm{C}$. On the fourth day it fell to $37^{\circ} \mathrm{C}$., and remained low with slight variation for five days. On the tenth day the temperature was $36^{\circ} \mathrm{C}$., and the bull died on the eleventh day. The animal had fed well until two days before death, when the appetite failed and the bull remained lying down. There were no symptoms of rinderpest. The lungs, liver, and spleen contained numerous cysts, and there was congestion of the mucous membrane of the fourth stomach and small intestines. The Peyer's patches were raised and congested.

During the year I observed about thirty cases of a more or less similar kind, where the symptoms of rinderpest were suppressed, and few lesions beyond the presence of numerous cysts to be found after death.

\section{DISTOMATOSIS.}

This disease is known to be prevalent among cattle grazing in marshy land subject to inundations. Pease has given a description of the disease as met with among India cattle, and of the parasite, the fasciola hepatica. In its relation to rinderpest exactly similar observations have been made as described in cattle affccted with hydatids. The temperature seldom rises, tends to go below normal, and the animal dies in the early stage, or, if it lives a longer period, no marked symptoms of rinderpest appear. At the post-mortem it is often difficult to say what the cause of death has been. Beyond the 
cirrhotic liver, congestion of the fourth stomach and intestines to a greater or less degree, and sometimes a bile-stained appearance of the tissues, there were no changes of sufficient severity to account for death.

Animals affected with hydatids often remain in apparent health and good condition, but those suffering from distomatosis are generally emaciated and anamic, skin dry, and bowels irregular, and this state renders them more susceptible to the attack of any other disease.

\title{
APHTHA.
}

During the winter months a contagious form of aphtha frequently makes its appearance among the cattle kept at the Laboratory for experimental use. The infection is brought by the hill cattle coming from the parts some 60 to 80 miles further in the Himalayas. The disease affects the muzzle, angles of the mouth, mucous surface of gums, lips, under surface of the tongue, and floor of the mouth. It is contagious and spreads very rapidly among the whole herd. It causes no elevation of temperature, and only affects the animal in so far that the lesions of the mouth render feeding painful, with a consequent loss of condition. The sores heal and the effect of the disease passes over in about three weeks. In the first stage vesicles are formed which exude a serous fluid, and these change into ulcers which become covered by a crust and take several days to heal. Animals suffering from this form of a phtha are less resistant to an attack of rinderpest, owing to their debilitated state. It is also frequently difficult to distinguish between the aphthous ulcers and those of rinderpest, when situated in the mucous membrane of the mouth. The ulcers of aphtha tend to run together into a circular lesion about the size of a threepenny piece or a sixpence. They are also covered with a necrotic crust. In rinderpest the ulcers are clear-cut and pin-point in appearance, do not run together, and are not covered with crust.

\section{DESCRIPTION OF PLATE VIII.}

a. Trypanosomata found in blood of cattle.

b. Forms of the same in smears from the spleen.

c. Piroplasmata in red blood corpuscles.

Drawn with Zeiss oil-immersion apochromatic $2 \mathrm{~mm}$. lens. Compensating ocular 8. Magnification 1000 diameters.

\section{THE PROPHYLACTIC TREATMENT OF RINDERPEST BY MEANS OF PREVENTIVE INOCULATION, MORE ESPECIALLY CONSIDERED IN REGARD TO THE CONDITIONS PREVAILING IN INDIA.}

\author{
By Captain G. K. WAIKER, F.R.C.V.S., Indian Civil Veterinary \\ Department.
}

IT may be well, at the outset, to say a few words with regard to the peculiar conditions appertaining to the treatment of cattle disease in India. It is a matter of fairly common knowledge that cattle are popularly regarded as sacred in that country. This assertion serves 